\title{
Curability of Leprosy.
}

(Comment on Dr. Rose's Article published in 'Leprosy Revierw.' Vol. V. No. 4.)

J. Rodriguez.

7 Editor of LEPROSY REVIEw has asked me to comment on a paper with the above title which appeared in LEPROSY REVIEW recently.

Dr. Rose is certainly to be congratulated on the fair and moderate way in which he has discussed his data. $\mathrm{He}$ seems to have taken full advantage of the unusual opportunities found in British Guiana to do what many other workers would like to see done more generally, that is, a really adequate follow-up of paroled or discharged quiescent and arrested cases. We are finding out that in leprosy, as in other chronic infectious diseases, our criteria as to the cure should not be based solely on the condition of the patient at the time of discharge from the hospital, but that the subsequent history should also be taken into consideration as well.

As I have not been able to follow-up our cases after parole as thoroughly as Dr. Rose did, I am not in a position at the present time to either confirm or refute his basic findings. Wherever there have been a considerable number of paroled patients, great difficulty has been found in following up cases. In the province of Cebu, Philippine Islands, there have been paroled from Culion and from the local Treatment Station, during the period from 1923-1934, 1,050 cases among a population estimated to be slightly over 1,000,000. A number of them are living in distant islands and in inaccessible mountainous regions. We have been trying to keep track of as many of them as possible and we expect to be able to present data on them somewhat along the lines of Dr. Rose's work in the near future. Up to the present, we already have on our records 21 cases which have relapsed after being quiescent and arrested for from 6 to $10 \frac{1}{2}$ years. Also, in Cebu we have had the opportunity of observing five paroled cases who have lived under almost ideal conditions as regards hygiene, food and treatment after discharge from the station. Unfortunately, four of them relapsed after varying periods ranging from four months to three years.

Coming now to a couple of details regarding the article. of Dr. Rose, quite a few workers would probably object to. the use of the word " recovered " in the manner suggested. 
by him. The four cases mentioned above were absolutely free from the slightest mark or blemish at the time of parole and should perhaps be classified as "quiescent and recovered " in accordance with Dr. Rose's classification, but as we now know, they had not quite recovered because definite cutaneous lesions developed on them subsequently. It is desirable perhaps to select another word to indicate the feature that Dr. Rose wants to emphasise.

Dr. Rose's statement that " cases treated in early childhood, moreover, yield more readily to treatment than the adult " (last sentence, page 155), seems to be directly contradictory to the opinion of D. E. B. Christian, who says in his last article (Leprosy in India, October, 1934, last sentence, page 195), "In children leprosy treatment does not give such quick results as in adults, but when they reach adult age they improve and clear up rapidly under treatment." It is striking that two good leprologists, using apparently the same method of treatment and the same criteria for testing the effects of a cure (frequently of relapses), should arrive at diametrically opposite conclusions in the effect of the treatment in the same class of patients (children). As has been stated in one of our last articles, such results are baffling and confusing to the other workers.

It may be possible for many to offer criticisms regarding some details in Dr. Rose's article, but very few workers are in a position to make fundamental criticisms, because there are so few, if any, who have been able to do what he has accomplished. Perhaps his best critic will be the ultimate results of his own work. He concludes his paper thus :

"When this (children's home) has been completed, we shall have as effective a protection from leprosy as exists in any country in which leprosy is indigenous." We should be able to see in the next two or three decades whether the protection has been really adequate or not.

I should like to take this opportunity to clarify my position with regard to the general outlook for the early macular (?) cases. I have been much distressed lest due to lack of clarity in my last two articles appearing in the Review, I have given the impression that the outlook for such cases is particularly gloomy. This seems to be hinted in the following statement appearing in the editorial in the October, 1934, issue of the REVIEw :

" The majority of Dr. Rose's cases appear to be those in which the bacilli on the recognised acid-fast forms could be detected, and it is just there that Dr. Rodriguez appeared to hold out the most hope." 
While this is certainly correct, it must be emphasised that it is true only in so far as the chaulmoogra treatment is concerned. The title of my article referred to was " Results of the Chaulmoogra Treatment in Very Early Cases of Leprosy." I must hasten to add that from a general view point, however, my experience has been that as a whole, the outlook for the average early macular leprosy is certainly much brighter than that of the average early cutaneous case. To begin with, he stands a good chance of becoming arrested without the years of painful injections. We hope to be able to present figures shortly which tend to prove that at least among the children of lepers, the incidence becoming positive, i.e., progressing to the cutaneous stage, was much reduced after they had been placed in hygienic surroundings and given adequate diet and care, irrespective of whether chaulmoogra treatment was given or not. In other words, we are fully in accord with Cochrane when he says: (International Journal of Leprosy, October-December, 1934, page 388) :

"We have no evidence that the present antileprosy drugs can prevent the development of further lesions in such cases (children with inactive, very early or abortive lesions) ... From this, it would seem that proper living conditions constitute our main defence in such cases.'

The point we have tried to emphasise in our article was that in precisely those cases referred to by Cochrane, the chaulmoogra derivatives appear to be of questionable value and may even prove harmful, since it has been shown by Read in his work on dogs, that the kidneys may be damaged by too large doses or too prolonged injections.

Manifestly, it is unfair to bring such cases to a home, give them good food, adequate care, administer to them chaulmoogra injections, and then give all the credit to the injections. We believe that if we wish really to evaluate the effects of the chaulmoogra treatment in such cases, we should have controls consisting of children given exactly the same care as the others, but receiving either no injections at all or only a trivial number of them.

Furthermore, the criteria to be used in judging the effect of the treatment should not be based merely on the changes in the appearance or nature of the macular lesions, but principally on whether the development of bacteriologically positive cutaneous lesions has been prevented or not.

While the publication of unreasonable and unduly pessimistic reports regarding the effects of the chaulmoogra treatment certainly to be deplored on account of its 
unfavourable effects on prophylactic measures against the disease, it is believed that far greater harm will be done to the cause if leprosy workers do not adopt a more critical and rigid attitude towards their results. As it is, a great deal of harm already has been done to the chaulmoogra treatment by well-intentioned but over-enthusiastic and probably not over-critical workers, who would have us all agree that chaulmoogra oil has been proven definitely to be a specific in leprosy. Not a few even seem to believe that leprosy can be eradicated by indiscriminate injection of " all and sundry, especially in out-patient centres." Perhaps it were better if we admit that although this drug is certainly useful in the treatment of leprosy, " there is still much room for improvement with regard to our knowledge as to its proper indications, limitations and action in the human body," and that therefore it is not yet safe to give undue weight to the value of this particular treatment in schemes to control the disease.

Finally, I would like to state that the above opinions are entirely personal and should not be considered as reflecting in any way the opinions of co-workers in the Philippines. 